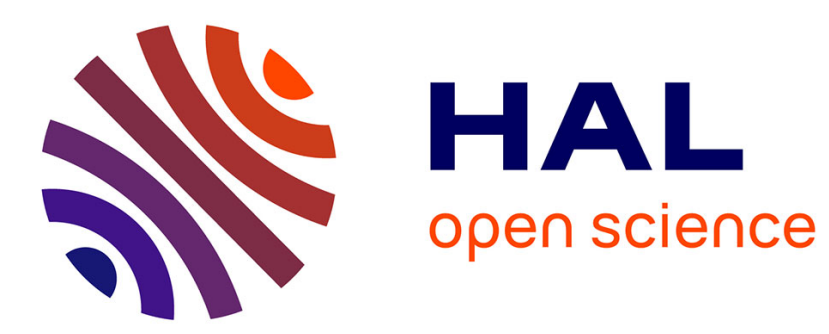

\title{
Analysis of robustness of homogeneous systems with time delays using Lyapunov-Krasovskii functionals
}

Denis Efimov, Alexander Aleksandrov

\section{To cite this version:}

Denis Efimov, Alexander Aleksandrov. Analysis of robustness of homogeneous systems with time delays using Lyapunov-Krasovskii functionals. International Journal of Robust and Nonlinear Control, In press, 10.1002/rnc.5115 . hal-02614951

\section{HAL Id: hal-02614951 \\ https://hal.inria.fr/hal-02614951}

Submitted on 21 May 2020

HAL is a multi-disciplinary open access archive for the deposit and dissemination of scientific research documents, whether they are published or not. The documents may come from teaching and research institutions in France or abroad, or from public or private research centers.
L'archive ouverte pluridisciplinaire HAL, est destinée au dépôt et à la diffusion de documents scientifiques de niveau recherche, publiés ou non, émanant des établissements d'enseignement et de recherche français ou étrangers, des laboratoires publics ou privés. 


\title{
Analysis of robustness of homogeneous systems with time delays using Lyapunov-Krasovskii functionals
}

\author{
D. Efimov, A. Aleksandrov
}

\begin{abstract}
The paper is devoted to stability analysis of homogeneous time-delay systems applying the Lyapunov-Krasovskii theory, and a generic structure of the functional is given that suits for any homogeneous system of non-zero degree (and can also be used for any dynamics admitting a homogeneous approximation). The obtained stability conditions are utilized to evaluate the domain of attraction for the delayed twisting control algorithm.
\end{abstract}

\section{INTRODUCTION}

The influence of delays on the system stability and performance is important for many engineering systems [1], [2], [3]. Design of control and estimation algorithms, which are robust with respect to uncertain and time-varying delays, is a popular topic of the control theory [4]. The most of existing solutions deal with linear time-delay models, which is originated by complexity of stability analysis for the nonlinear case. Indeed, constructive variants of selection of a Lyapunov-Krasovskii functional or a Lyapunov-Razumikhin function are elaborated for linear systems (or close to them nonlinear dynamics) [5], but for generic scenario it is rare to find an applicable recommendation.

Homogeneous dynamical systems take an intermediate place between linear and nonlinear ones [6]. The theory of homogeneous dynamical systems has been proposed for ordinary differential equations [7], [8], [9], [10], differential inclusions [11], [6], time-delay systems [12], [13] and partial differential equations [14]. The main feature of a homogeneous nonlinear system is that its local behavior is the same as the global one, the homogeneous stable/unstable systems admit homogeneous Lyapunov/Chetaev functions [10], [15], [16], [17], [18] and possess robustness properties with respect to external inputs and delays [19], [13], [20]. Since such a subclass of nonlinear systems (having global stability or convergence) is rather small, the concept of local homogeneity has been introduced [10], [21], [16], [22].

In [23], [24], [25], [26], [27], [13], [20] it has been shown that homogeneous systems have certain robustness of stability with respect to delays, e.g., if they are globally asymptotically stable for some delay with non-zero degree, then they preserve this property independently of delay (IOD). For the case of positive degree it has been proven that global asymptotic stability in the delay-free case implies local asymptotic stability for any value of delay. For the case of negative degree, it is shown that if the system is globally asymptotically stable in the delay-free case, then for any delay it is globally asymptotically stable with respect to a compact set containing the origin. The latter results have been obtained in [13], [20] using Lyapunov-Razumikhin theory.

In the present work, first, following the framework of [28], the same results are obtained by proposing a generic structure Lyapunov-Krasovskii functional for any homogeneous system with non-zero degree being asymptotically stable in the delayfree case. The presented approach allows the domain of stability/attraction to be evaluated. Second, the proposed LyapunovKrasovskii functional is used to estimate the domain of convergence for a delayed twisting algorithm.

The outline of this paper is as follows. The preliminary definitions and the homogeneity for time-delay systems are given in Section II. The main result is presented in Section III. An example is considered in Section IV.

D. Efimov is with Inria, Univ. Lille, CNRS, UMR 9189 - CRIStAL, F-59000 Lille, France and ITMO University, 49 av. Kronverkskiy, 197101 Saint Petersburg, Russia.

A. Aleksandrov is with Saint Petersburg State University, 7-9 Universitetskaya nab., 199034 Saint Petersburg, Russia.

This work was partially supported by the Government of Russian Federation (Grant 08-08), by the Ministry of Science and Higher Education of Russian Federation, passport of goszadanie no. 2019-0898, and by the Russian Foundation for Basic Research (grant no. 19-01-00146-a). 


\section{PReliminaries}

Consider an autonomous functional differential equation of retarded type [3]:

$$
d x(t) / d t=f\left(x_{t}\right), t \geq 0,
$$

where $x(t) \in \mathbb{R}^{n}$ and $x_{t} \in C_{[-\tau, 0]}$ is the state function, $x_{t}(s)=x(t+s),-\tau \leq s \leq 0$ (we denote by $C_{[-\tau, 0]}$ the Banach space of continuous functions $\phi:[-\tau, 0] \rightarrow \mathbb{R}^{n}$ with the uniform norm $\|\phi\|=\sup _{-\tau \leq \varsigma \leq 0}|\phi(\varsigma)|$, where $|\cdot|$ is the standard Euclidean norm); $f: C_{[-\tau, 0]} \rightarrow \mathbb{R}^{n}$ ensures existence and uniqueness of solutions in forward time (such a requirement can be imposed even for discontinuous time-delay systems [29], [30]), $f(0)=0$. For the system (1), denote the solution that corresponds to the initial functional condition $x_{0} \in C_{[-\tau, 0]}$ by $x\left(t, x_{0}\right)$ and the restriction of the solution on the interval $[t-\tau, t]$ by $x_{t}^{x_{0}}$ (then $x_{t}^{x_{0}}(s)=x\left(t+s, x_{0}\right)$ for $\left.-\tau \leq s \leq 0\right)$, which is defined on time interval $[-\tau, T)$ with $T \in \mathbb{R}_{+} \cup\{+\infty\}$. The representation (1) includes pointwise or distributed time-delay systems.

The upper right-hand Dini derivative of a locally Lipschitz continuous functional $V: C_{[-\tau, 0]} \rightarrow \mathbb{R}$ along the system (1) solutions is defined as follows for any $\phi \in C_{[-\tau, 0]}$ :

$$
D^{+} V(\phi)=\lim \sup _{h \rightarrow 0^{+}} \frac{V\left(\phi_{h}\right)-V(\phi)}{h}
$$

where $\phi_{h} \in C_{[-\tau, 0]}$ for $0<h<\tau$ is given by

$$
\phi_{h}= \begin{cases}\phi(\theta+h), & \theta \in[-\tau,-h) \\ \phi(0)+f(\phi)(\theta+h), & \theta \in[-h, 0] .\end{cases}
$$

A continuous function $\sigma: \mathbb{R}_{+} \rightarrow \mathbb{R}_{+}$belongs to class $\mathcal{K}$ if it is strictly increasing and $\sigma(0)=0$; it belongs to class $\mathcal{K}_{\infty}$ if it is also unbounded. A continuous function $\beta: \mathbb{R}_{+} \times \mathbb{R}_{+} \rightarrow \mathbb{R}_{+}$belongs to class $\mathcal{K} \mathcal{L}$ if $\beta(\cdot, r) \in \mathcal{K}$ and $\beta(r, \cdot)$ is decreasing to zero for any fixed $r>0$. The symbol $\overline{1, m}$ is used to denote a sequence of integers $1, \ldots, m$.

\section{A. Stability definitions}

Let $\mathcal{A} \subset C_{[-\tau, 0]}$ be a bounded set, it is called forward invariant for (1) if $x_{0} \in \mathcal{A}$ implies that $x_{t}^{x_{0}} \in \mathcal{A}$ for all $t \geq 0$, denote distance to the set as $\|x\|_{\mathcal{A}}=\inf _{y \in \mathcal{A}}\|x-y\|$. Let $\Omega \subset C_{[-\tau, 0]}$ be an open neighborhood of a forward invariant set $\mathcal{A}$.

Definition 1. The system (1) is said to be

(a) stable at $\mathcal{A}$ in $\Omega$ if there is $\sigma \in \mathcal{K}$ such that for any $x_{0} \in \Omega$, the solution $x_{t}^{x_{0}}$ is defined and $\left\|x_{t}^{x_{0}}\right\|_{\mathcal{A}} \leq \sigma\left(\left\|x_{0}\right\|_{\mathcal{A}}\right)$ for all $t \geq 0$;

(b) asymptotically stable at $\mathcal{A}$ in $\Omega$ if it is stable in $\Omega$ and $\lim _{t \rightarrow+\infty}\left\|x_{t}^{x_{0}}\right\|_{\mathcal{A}}=0$ for any $x_{0} \in \Omega$.

If $\Omega=C_{[-\tau, 0]}$, then the corresponding properties are called global stability/asymptotic stability of (1) at $\mathcal{A}$.

For $\mathcal{A}=\{0\}$ the properties given in this definition are reduced to the standard ones [4] (see also the concept of ultimate boundedness in [2]).

\section{B. Homogeneity}

For any $r_{i}>0, i=\overline{1, n}$ and $\lambda>0$, define the dilation matrix $\Lambda_{r}(\lambda)=\operatorname{diag}\left\{\lambda^{r_{i}}\right\}_{i=1}^{n}$ and the vector of weights $\mathbf{r}=$ $\left[r_{1}, \ldots, r_{n}\right]^{\top}, r_{\max }=\max _{1 \leq j \leq n} r_{j}$ and $r_{\min }=\min _{1 \leq j \leq n} r_{j}$.

For any $r_{i}>0, i=\overline{1, n}$ and $x \in \mathbb{R}^{n}$ the homogeneous norm can be defined, for example, as follows

$$
|x|_{r}=\left(\sum_{i=1}^{n}\left|x_{i}\right|^{\rho / r_{i}}\right)^{1 / \rho}, \rho \geq r_{\max } .
$$

For all $x \in \mathbb{R}^{n}$, its Euclidean norm $|x|$ is related with the homogeneous one:

$$
\underline{\sigma}_{r}\left(|x|_{r}\right) \leq|x| \leq \bar{\sigma}_{r}\left(|x|_{r}\right)
$$


for some $\underline{\sigma}_{r}, \bar{\sigma}_{r} \in \mathcal{K}_{\infty}$ [18]. The homogeneous norm has an important property that is $\left|\Lambda_{r}(\lambda) x\right|_{r}=\lambda|x|_{r}$ for all $x \in \mathbb{R}^{n}$. Define $\mathbb{S}_{r}=\left\{x \in \mathbb{R}^{n}:|x|_{r}=1\right\}$.

For any $r_{i}>0, i=\overline{1, n}$ and $\phi \in C_{[-\tau, 0]}$ the homogeneous norm can be defined as follows

$$
\|\phi\|_{r}=\left(\sum_{i=1}^{n}\left\|\phi_{i}\right\|^{\rho / r_{i}}\right)^{1 / \rho}, \rho \geq r_{\max } .
$$

There exist two functions $\underline{\rho}_{r}, \bar{\rho}_{r} \in \mathcal{K}_{\infty}$ such that for all $\phi \in C_{[-\tau, 0]}[12]$ :

$$
\underline{\rho}_{r}\left(\|\phi\|_{r}\right) \leq\|\phi\| \leq \bar{\rho}_{r}\left(\|\phi\|_{r}\right)
$$

The homogeneous norm in the Banach space has the same important property that $\left\|\Lambda_{r}(\lambda) \phi\right\|_{r}=\lambda\|\phi\|_{r}$ for all $\phi \in C_{[-\tau, 0]}$. In $C_{[-\tau, 0]}$ the corresponding unit sphere is $\mathcal{S}_{r}=\left\{\phi \in C_{[-\tau, 0]}:\|\phi\|_{r}=1\right\}$. Define $B_{\rho}^{\tau}=\left\{\phi \in C_{[-\tau, 0]}:\|\phi\|_{r} \leq \rho\right\}$ as a closed ball of radius $\rho>0$ in $C_{[-\tau, 0]}$.

Definition 2. [31] The function $g: C_{[-\tau, 0]} \rightarrow \mathbb{R}$ is called $\mathbf{r}$-homogeneous if for any $\phi \in C_{[-\tau, 0]}$ the relation

$$
g\left(\Lambda_{r}(\lambda) \phi\right)=\lambda^{d} g(\phi)
$$

holds for some $d \in \mathbb{R}$ and all $\lambda>0$.

The function $f: C_{[-\tau, 0]} \rightarrow \mathbb{R}^{n}$ is called $\mathbf{r}$-homogeneous if for any $\phi \in C_{[-\tau, 0]}$ the relation

$$
f\left(\Lambda_{r}(\lambda) \phi\right)=\lambda^{d} \Lambda_{r}(\lambda) f(\phi)
$$

holds for some $d \geq-r_{\min }$ and all $\lambda>0$.

In both cases, the constant $d$ is called the degree of homogeneity.

The introduced notion of weighted homogeneity in $C_{[-\tau, 0]}$ is reduced to the standard one in $\mathbb{R}^{n}$ if $\tau \rightarrow 0$.

An advantage of homogeneous systems described by ordinary differential equations is that any its solution can be obtained from another solution under the dilation and a suitable time re-parametrization. A similar property holds for functional homogeneous systems:

Proposition 1. [13] Let $x\left(t, x_{0}\right)$ be a solution of the $\mathbf{r}$-homogeneous system (1) with the degree $d$ for an initial condition $x_{0} \in C_{[-\tau, 0]}, \tau \in(0,+\infty)$. For any $\lambda>0$ the functional differential equation

$$
d y(t) / d t=f\left(y_{t}\right), t \geq 0
$$

with $y_{t} \in C_{\left[-\lambda^{-d} \tau, 0\right]}$, has a solution $y\left(t, y_{0}\right)=\Lambda_{r}(\lambda) x\left(\lambda^{d} t, x_{0}\right)^{1}$ for all $t \geq 0$ with the initial condition $\left.y_{0} \in C_{[-\lambda-d} \tau, 0\right]$, $y_{0}(s)=\Lambda_{r}(\lambda) x_{0}\left(\lambda^{d} s\right)$ for $s \in\left[-\lambda^{-d} \tau, 0\right]$.

The following results have also been obtained in [13], [20]:

Lemma 1. [13] Let the system (1) be $\mathbf{r}$-homogeneous with degree $d \neq 0$ and globally asymptotically stable at the origin for some delay $0<\tau_{0}<+\infty$, then it is globally asymptotically stable at the origin IOD.

For the next two results we assume that $f$ in (1) is a uniformly continuous function.

Lemma 2. [13] Let $f\left(x_{t}\right)=F(x(t), x(t-\tau))$ in (1) and the system (1) be $\mathbf{r}$-homogeneous with degree $d>0$ and globally asymptotically stable at the origin for $\tau=0$, then for any $\rho>0$ there is $0<\tau_{0}<+\infty$ such that (1) is asymptotically stable at the origin in $B_{\rho}^{\tau}$ for any delay $0 \leq \tau \leq \tau_{0}$.

Lemma 3. [20] Let $f\left(x_{t}\right)=F(x(t), x(t-\tau))$ in (1) and the system (1) be $\mathbf{r}$-homogeneous with degree $d<0$ and globally asymptotically stable at the origin for $\tau=0$, then for any $\rho>0$ there is $0<\tau_{0}<+\infty$ such that (1) is globally asymptotically stable at $B_{\rho}^{\tau}$ for any delay $0 \leq \tau \leq \tau_{0}$.

${ }^{1}$ If time is scaled $t \rightarrow \lambda^{d} t$ then the argument of $f: C_{[-\tau, 0]} \rightarrow \mathbb{R}^{n}$ in (1) is also scaled to $f: C_{\left[-\lambda^{-} \tau_{\tau, 0]}\right.} \rightarrow \mathbb{R}^{n}$ in (2). 
Thus, (1) is locally robustly stable with respect to a sufficiently small delay if it is $\mathbf{r}$-homogeneous and stable in the delay-free case. In [13], [20], the Lyapunov-Razumikhin approach was used to prove these results.

Finally, Young's inequality claims that for any $\mathfrak{a}, \mathfrak{b} \in \mathbb{R}_{+}$and $\gamma>0, \delta>0$ :

$$
\mathfrak{a}^{\gamma} \mathfrak{b}^{\delta} \leq \frac{1}{p} \mathfrak{a}^{\gamma p}+\frac{p-1}{p} \mathfrak{b}^{\frac{\delta p}{p-1}}
$$

for any $p>1$, while Hölder's inequality for any $f, g: I \rightarrow \mathbb{R}$ with $I \subset \mathbb{R}$ ensures that

$$
\int_{I}|f(s) g(s)| d s \leq\left(\int_{I}|f(s)|^{p} d s\right)^{\frac{1}{p}}\left(\int_{I}|g(s)|^{\frac{p}{p-1}} d s\right)^{\frac{p-1}{p}}
$$

for any $p>1$. Another related result can be obtained using the properties of homogeneous functions and a specially defined homogeneous norm:

Lemma 4. Let $\mathfrak{a}, \mathfrak{b} \in \mathbb{R}_{+}$and $\ell>0, \alpha>0, \beta>0, \gamma>0, \delta>0$ be given, then

$$
\mathfrak{a}^{\alpha}+\mathfrak{b}^{\beta}-\ell \mathfrak{a}^{\gamma} \mathfrak{b}^{\delta} \geq 0
$$

provided that

1) $\max \left\{\mathfrak{a}^{\alpha}, \mathfrak{b}^{\beta}\right\} \geq \ell^{\frac{1}{1-\frac{\gamma}{\alpha}-\frac{\delta}{\beta}}}$ and $\frac{\gamma}{\alpha}+\frac{\delta}{\beta}<1$,

2) $\max \left\{\mathfrak{a}^{\alpha}, \mathfrak{b}^{\beta}\right\} \leq \ell^{\frac{1}{1-\frac{\gamma}{\alpha}-\frac{\delta}{\beta}}}$ and $\frac{\gamma}{\alpha}+\frac{\delta}{\beta}>1$.

Proof. Define weights $r_{1}=\frac{1}{\alpha}$ and $r_{2}=\frac{1}{\beta}$ and a corresponding homogeneous norm

$$
|(\mathfrak{a}, \mathfrak{b})|_{r}=\max \left\{\mathfrak{a}^{\alpha}, \mathfrak{b}^{\beta}\right\}
$$

then the scalar functions $\mathfrak{a}^{\alpha}+\mathfrak{b}^{\beta}$ and $\mathfrak{a}^{\gamma} \mathfrak{b}^{\delta}$ are $\mathbf{r}$-homogeneous (with $\mathbf{r}=\left[r_{1}, r_{2}\right]^{\top}$ ) of degrees 1 and $\frac{\gamma}{\alpha}+\frac{\delta}{\beta}$, respectively. Therefore, the following inequality is satisfied for $\mathfrak{a}, \mathfrak{b} \in \mathbb{R}_{+}$:

$$
\mathfrak{a}^{\gamma} \mathfrak{b}^{\delta} \leq s|(\mathfrak{a}, \mathfrak{b})|_{r}^{\frac{\gamma}{\alpha}+\frac{\delta}{\beta}}
$$

where

$$
s=\sup _{|(\mathfrak{a}, \mathfrak{b})|_{r}=1} \mathfrak{a}^{\gamma} \mathfrak{b}^{\delta}=1
$$

then the property

$$
\mathfrak{a}^{\alpha}+\mathfrak{b}^{\beta}-\ell \mathfrak{a}^{\gamma} \mathfrak{b}^{\delta} \geq 0
$$

for any $\mathfrak{a}, \mathfrak{b} \in \mathbb{R}_{+}$and $c>0, \alpha>0, \beta>0, \gamma>0, \delta>0$ is verified provided that

$$
|(\mathfrak{a}, \mathfrak{b})|_{r}-\ell|(\mathfrak{a}, \mathfrak{b})|_{r}^{\frac{\gamma}{\alpha}+\frac{\delta}{\beta}} \geq 0
$$

Hence, if

$$
\frac{\gamma}{\alpha}+\frac{\delta}{\beta}<1
$$

then this inequality is satisfied for all $|(\mathfrak{a}, \mathfrak{b})|_{r} \geq \ell^{\frac{1}{1-\frac{\gamma}{\alpha}-\frac{\delta}{\beta}}}$, and if

$$
\frac{\gamma}{\alpha}+\frac{\delta}{\beta}>1
$$

then for $|(\mathfrak{a}, \mathfrak{b})|_{r} \leq \ell^{\frac{1}{1-\frac{\gamma}{\alpha}-\frac{\delta}{\beta}}}$.

\section{MAIN RESULTS}

Consider a variant of the system (1):

$$
\dot{x}(t)=F(x(t), x(t-\tau)),
$$


where $F: \mathbb{R}^{2 n} \rightarrow \mathbb{R}^{n}$ is differentiable with respect to the first argument, and introduce the following hypotheses:

Assumption 1. The system (3) is $\mathbf{r}$-homogeneous of degree $\nu \geq-r_{\min }$, i.e., for all $\lambda>0, x \in \mathbb{R}^{n}$ and $z \in \mathbb{R}^{n}$ :

$$
F\left(\Lambda_{r}(\lambda) x, \Lambda_{r}(\lambda) z\right)=\lambda^{\nu} \Lambda_{r}(\lambda) F(x, z)
$$

and

$$
\sup _{|\xi|_{r} \leq 1,|\zeta|_{r} \leq 1}|F(\xi, \zeta)|<+\infty, \sup _{|\xi|_{r} \leq 1,|\zeta|_{r} \leq 1}\left|\frac{\partial F(\xi, \zeta)}{\partial \xi}\right|<+\infty .
$$

Assumption 2. The system (3) is globally asymptotically stable at the origin if $\tau=0$.

Under these assumptions [10], [15], there is a twice continuously differentiable Lyapunov function $V: \mathbb{R}^{n} \rightarrow \mathbb{R}_{+}$for (3), which can be selected to be $\mathbf{r}$-homogeneous of degree $\mu \geq-\nu$, then for all $x \in \mathbb{R}^{n}$ :

$$
\begin{gathered}
V\left(\Lambda_{r}(\lambda) x\right)=\lambda^{\mu} V(x) \quad \forall \lambda>0, \\
c_{1}|x|_{r}^{\mu} \leq V(x) \leq c_{2}|x|_{r}^{\mu}, \\
\frac{d V(x)}{d x} F(x, x) \leq-\varsigma|x|_{r}^{\nu+\mu}
\end{gathered}
$$

for some $c_{1}>0, c_{2}>0$ and $\varsigma>0$.

Remark 1. If the system (3) is $\mathbf{r}$-homogeneous with degree $\nu$, then a direct computation shows that it is also $\tilde{\mathbf{r}}$-homogeneous for $\tilde{\mathbf{r}}=r_{\max }^{-1}\left(r_{1}, \ldots, r_{n}\right)^{T}$ with degree $\tilde{\nu}=\frac{\nu}{r_{\max }}$. Hence, without loosing generality in the following we will assume that $r_{\max }=1$.

To proceed we need the following auxiliary result:

Lemma 5. Let Assumption 1 be satisfied, then for any twice continuously differentiable and $\mathbf{r}-$ homogeneous of degree $\mu \geq 2$ function $V: \mathbb{R}^{n} \rightarrow \mathbb{R}_{+}$:

$$
\begin{gathered}
\left|\frac{d V(x)}{d x} F(x, z)\right| \leq w \max \left\{|x|_{r}^{\mu-1}|z|_{r}^{\nu+1},|x|_{r}^{\mu+\nu}\right\}, \\
\left|F(x, q)^{\top} \frac{d^{2} V(x)}{d x^{2}} F(x, z)\right| \leq v \max \left\{|x|_{r}^{\mu+2 \nu},|x|_{r}^{\mu-2}|z|_{r}^{2 \nu+2},|x|_{r}^{\mu-2}|q|_{r}^{2 \nu+2}\right\}, \\
\left|\frac{d V(x)}{d x} \frac{\partial F(x, z)}{\partial x} F(x, q)\right| \leq u \max \left\{|x|_{r}^{\mu+2 \nu},|x|_{r}^{\mu-1}|z|_{r}^{2 \nu+1},|x|_{r}^{\mu-1}|q|_{r}^{2 \nu+1}\right\},
\end{gathered}
$$

where

$$
\begin{gathered}
w=\sup _{y \in \mathbb{S}_{r}}\left|\frac{d V(y)}{d y}\right| \sup _{|\xi|_{r} \leq 1,|\zeta|_{r} \leq 1}|F(\xi, \zeta)|, \\
v=\sup _{y \in \mathbb{S}_{r}}\left|\frac{d^{2} V(y)}{d y^{2}}\right| \sup _{|\xi|_{r} \leq 1,|\zeta|_{r} \leq 1}|F(\xi, \zeta)|^{2}, \\
u=\sup _{y \in \mathbb{S}_{r}}\left|\frac{d V(y)}{d y}\right| \sup _{|\xi|_{r} \leq 1,|\zeta|_{r} \leq 1}\left|\frac{\partial F(\xi, \zeta)}{\partial \xi} F(\xi, \zeta)\right| .
\end{gathered}
$$

Proof. To substantiate the first property define $\lambda=\max \left\{|z|_{r},|x|_{r}\right\}$, then using homogeneity of $V$ and $F$ we obtain

$$
\left|\frac{d V(x)}{d x} F(x, z)\right|=|x|_{r}^{\mu} \lambda^{\nu}\left|\frac{d V(y)}{d y} \Lambda_{r}^{-1}\left(|x|_{r}\right) \Lambda_{r}(\lambda) F(\xi, \zeta)\right|
$$

for $y=\Lambda_{r}^{-1}\left(|x|_{r}\right) x, \xi=\Lambda_{r}^{-1}(\lambda) x$ and $\zeta=\Lambda_{r}^{-1}(\lambda) z$. Using the diagonal structure of the matrix $\Lambda_{r}^{-1}\left(|x|_{r}\right) \Lambda_{r}(\lambda)$ we get:

$$
\left|\frac{d V(x)}{d x} F(x, z)\right| \leq w|x|_{r}^{\mu} \lambda^{\nu}\left\{\begin{array}{ll}
\left(\frac{\lambda}{|x|_{r}}\right)^{r_{\max }} & |z|_{r}>|x|_{r} \\
1 & |z|_{r} \leq|x|_{r}
\end{array},\right.
$$

and since it has been imposed $r_{\max }=1$, we substantiated that

$$
\left|\frac{d V(x)}{d x} F(x, z)\right| \leq w \max \left\{|x|_{r}^{\mu-1}|z|_{r}^{\nu+1},|x|_{r}^{\mu+\nu}\right\} .
$$


For the second inequality define $y=\Lambda_{r}^{-1}\left(|x|_{r}\right) x, \xi=\Lambda_{r}^{-1}(\lambda) x, \theta=\Lambda_{r}^{-1}(\lambda) q$ and $\zeta=\Lambda_{r}^{-1}(\lambda) z$ for $\lambda=$ $\max \left\{|z|_{r},|q|_{r},|x|_{r}\right\}$, then by homogeneity:

$$
\begin{gathered}
\left|F(x, q)^{\top} \frac{d^{2} V(x)}{d x^{2}} F(x, z)\right|=|x|_{r}^{\mu} \lambda^{2 \nu}\left|F(\xi, \theta)^{\top} \Lambda_{r}(\lambda) \Lambda_{r}^{-1}\left(|x|_{r}\right) \frac{d^{2} V(y)}{d y^{2}} \Lambda_{r}^{-1}\left(|x|_{r}\right) \Lambda_{r}(\lambda) F(\xi, \zeta)\right| \\
\leq v|x|_{r}^{\mu} \lambda^{2 \nu} \begin{cases}\left(\frac{\lambda}{|x|_{r}}\right)^{2} & \max \left\{|z|_{r},|q|_{r}\right\}>|x|_{r} \\
1 & \max \left\{|z|_{r},|q|_{r}\right\} \leq|x|_{r}\end{cases}
\end{gathered}
$$

where $r_{\max }=1$ was substituted on the last step, then the desired property is proven:

$$
\begin{gathered}
\left|F(x, q)^{\top} \frac{d^{2} V(x)}{d x^{2}} F(x, z)\right| \leq v \max \left\{|x|_{r}^{\mu+2 \nu},|x|_{r}^{\mu-2} \lambda^{2 \nu+2}\right\} \\
=v \max \left\{|x|_{r}^{\mu+2 \nu},|x|_{r}^{\mu-2} \max \left\{|z|_{r}^{2 \nu+2},|q|_{r}^{2 \nu+2}\right\}\right\} \\
=v \max \left\{|x|_{r}^{\mu+2 \nu},|x|_{r}^{\mu-2}|z|_{r}^{2 \nu+2},|x|_{r}^{\mu-2}|q|_{r}^{2 \nu+2}\right\} .
\end{gathered}
$$

For the last inequality, again denote $y=\Lambda_{r}^{-1}\left(|x|_{r}\right) x, \xi=\Lambda_{r}^{-1}(\lambda) x, \theta=\Lambda_{r}^{-1}(\lambda) q$ and $\zeta=\Lambda_{r}^{-1}(\lambda) z$ for $\lambda=\max \left\{|z|_{r},|q|_{r},|x|_{r}\right\}$, then by homogeneity

$$
\left|\frac{d V(x)}{d x} \frac{\partial F(x, z)}{\partial x} F(x, q)\right|=\left|\frac{d V(y)}{d y} \Lambda_{r}^{-1}\left(|x|_{r}\right) \Lambda_{r}(\lambda) \frac{\partial F(\xi, \zeta)}{\partial \xi} F(\xi, \theta)\right||x|_{r}^{\mu} \lambda^{2 \nu},
$$

and once more using the diagonal structure of the matrix $\Lambda_{r}^{-1}\left(|x|_{r}\right) \Lambda_{r}(\lambda)$ we get:

$$
\begin{gathered}
\left|\frac{d V(x)}{d x} \frac{\partial F(x, z)}{\partial x} F(x, q)\right| \leq u|x|_{r}^{\mu} \lambda^{2 \nu} \begin{cases}\frac{\lambda}{|x|_{r}} & \max \left\{|z|_{r},|q|_{r}\right\}>|x|_{r} \\
1 & \max \left\{|z|_{r},|q|_{r}\right\} \leq|x|_{r}\end{cases} \\
\leq u \max \left\{|x|_{r}^{\mu+2 \nu},|x|_{r}^{\mu-1}|z|_{r}^{2 \nu+1},|x|_{r}^{\mu-1}|q|_{r}^{2 \nu+1}\right\}
\end{gathered}
$$

as required.

Note that $\nu \geq-r_{\min }$ and $r_{\min } \leq r_{\max }=1$ as we assumed (due to Remark 1), then for $\mu>2$ all powers in the obtained estimates are positive (the latter estimate for the case $\nu<0$ we can rewrite as $\max \left\{|x|_{r}^{\mu+2 \nu},|x|_{r}^{\frac{\mu}{2}-1}|z|_{r}^{2 \nu+\frac{\mu}{2}+1},|x|_{r}^{\frac{\mu}{2}-1}|q|_{r}^{2 \nu+\frac{\mu}{2}+1}\right\}$ since $|x|_{r}^{\frac{\mu}{2}}<\max \left\{|z|_{r}^{\frac{\mu}{2}},|q|_{r}^{\frac{\mu}{2}}\right\}$ in the latter two cases).

Now we are in position to formulate the main result:

Theorem 1. Let assumptions 1 and 2 hold for (3). Then for any value of the delay $\tau>0$ the properties

$$
W\left(x_{t}\right)>0, D^{+} W\left(x_{t}\right)<0
$$

are satisfied for $x_{t} \in \underline{\Omega}$ if $\nu<0$ or for $x_{t} \in \bar{\Omega}$ if $\nu>0$, where

$$
\begin{aligned}
W\left(x_{t}\right)= & V(x(t))+\left.b \int_{t-\tau}^{t}|x(s)|\right|_{r} ^{\rho} d s+g \int_{t-\tau}^{t}(s-t+\tau)|x(s)|_{r}^{\rho} d s \\
& +\int_{t-\tau}^{t} \frac{d V(x(t))}{d x} F(x(t), x(s)) d s,
\end{aligned}
$$

is a Lyapunov-Krasovskii functional for (3); $b>\frac{\tau}{\rho} \max \{4 v(\nu+1), u(\mu+4 \nu+2)\}, g>0$,

$$
\max \left\{\mu(\nu+1), 2(\nu+1) \frac{\mu+\nu}{\nu+2}, \frac{2\left(\nu+\frac{\mu}{2}\right)^{2}+\nu+\left(\frac{\nu}{2}+1\right) \mu}{\nu+1+\frac{\mu}{2}},(\nu+\mu) \frac{\frac{\mu}{2}+2 \nu+1}{\nu+\frac{\mu}{2}+1}\right\}<\rho<\nu+\mu,
$$

if $\nu<0$ or

$$
\nu+\mu<\rho<\min \left\{\mu(\nu+1), 2(\nu+1) \frac{\mu+\nu}{\nu+2}, \frac{(2 \nu+1)(\nu+\mu)}{\nu+1}\right\}
$$


if $\nu>0$, and $V$ is a twice continuously differentiable $\mathbf{r}$-homogeneous Lyapunov function for the case $\tau=0$ with degree

$$
\mu> \begin{cases}2 & \nu<0 \\ \nu+2 & \nu>0\end{cases}
$$

$\varsigma, c_{1}$ and $w, v, u$ are defined in (4) and Lemma 5, respectively;

$$
\begin{gathered}
\underline{\Omega}=\underline{\Omega}_{1} \cap \underline{\Omega}_{2}, \bar{\Omega}=\bar{\Omega}_{1} \cap \bar{\Omega}_{2}, \\
\underline{\Omega}_{1}=\left\{\phi \in C_{[-\tau, 0]}: \max \left\{|\phi(0)|_{r}^{\mu}, \int_{-\tau}^{0}|\phi(s)|_{r}^{\rho} d s\right\} \geq \underline{\varpi}_{1}\right\}, \\
\underline{\Omega}_{2}=\left\{\phi \in C_{[-\tau, 0]}: \max \left\{|\phi(0)|_{r}^{\nu+\mu}, \int_{-\tau}^{0}|\phi(s)|_{r}^{\rho} d s\right\} \geq \underline{\varpi}_{2}\right\}, \\
\bar{\Omega}_{1}=\left\{\phi \in C_{[-\tau, 0]}: \max \left\{|\phi(0)|_{r}^{\mu}, \int_{-\tau}^{0}|\phi(s)|_{r}^{\rho} d s\right\} \leq \varpi_{1}\right\}, \\
\bar{\Omega}_{2}=\left\{\phi \in C_{[-\tau, 0]}: \max \left\{|\phi(0)|_{r}^{\nu+\mu}, \int_{-\tau}^{0}|\phi(s)|_{r}^{\rho} d s\right\} \leq \bar{\varpi}_{2}\right\}
\end{gathered}
$$

where

$$
\begin{aligned}
& \underline{\varpi}_{1}=\max \left\{\left(\frac{w \tau}{\min \left\{c_{1}, b\right\}}\right)^{-\frac{\mu}{\nu}},\left(\frac{w \tau^{\frac{\rho-\nu-1}{\rho}}}{\min \left\{c_{1}, b\right\}}\right)^{\frac{1}{\mu}-\frac{\nu+1}{\rho}}\right\} \\
& \underline{\varpi}_{2}=\max \left\{\left(3 \frac{b+g \tau}{\min \{\varsigma, g\}}\right)^{\frac{\nu+\mu}{\nu+\mu-\rho}},\left(\frac{3 v \tau}{\min \{\varsigma, g\}}\right)^{-\frac{\nu+\mu}{\nu}},\left(3 v \frac{\rho-2(\nu+1)}{\rho \min \{\varsigma, g\}} \tau\right)^{\frac{(\rho-2(\nu+1))(\nu+\mu)}{(\nu+2) \rho-2(\nu+1)(\mu+\nu)}},\right.
\end{aligned}
$$

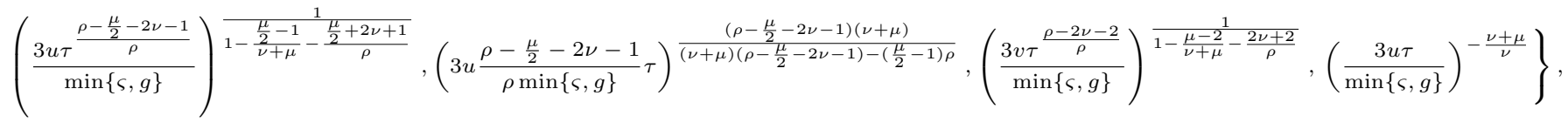

$$
\begin{aligned}
& \bar{\varpi}_{1}=\min \left\{\left(\frac{w \tau}{\min \left\{c_{1}, b\right\}}\right)^{-\frac{\mu}{\nu}},\left(\frac{w \tau^{\frac{\rho-\nu-1}{\rho}}}{\min \left\{c_{1}, b\right\}}\right)^{\frac{1}{\mu}-\frac{\nu+1}{\rho}}\right\} \\
& \bar{\varpi}_{2}=\min \left\{\left(3 \frac{b+g \tau}{\min \{\varsigma, g\}}\right)^{\frac{\nu+\mu}{\nu+\mu-\rho}},\left(\frac{3 v \tau}{\min \{\varsigma, g\}}\right)^{-\frac{\nu+\mu}{\nu}},\left(3 v \frac{\rho-2(\nu+1)}{\rho \min \{\varsigma, g\}} \tau\right)^{\frac{(\rho-2(\nu+1))(\nu+\mu)}{(\nu+2) \rho-2(\nu+1)(\mu+\nu)}},\right. \\
& \left.\left(\frac{3 u \tau^{\frac{\rho-2 \nu-1}{\rho}}}{\min \{\varsigma, g\}}\right)^{\frac{1}{\nu+\mu}-\frac{2 \nu+1}{\rho}},\left(3 u \frac{\rho-2 \nu-1}{\rho \min \{\varsigma, g\}} \tau\right)^{\frac{(\rho-2 \nu-1)(\nu+\mu)}{(\nu+1) \rho-(2 \nu+1)(\nu+\mu)}},\left(\frac{3 v \tau^{\frac{\rho-2 \nu-2}{\rho}}}{\min \{\varsigma, g\}}\right)^{\frac{1}{1-\frac{\mu-2}{\nu+\mu}-\frac{2 \nu+2}{\rho}}},\left(\frac{3 u \tau}{\min \{\varsigma, g\}}\right)^{-\frac{\nu+\mu}{\nu}}\right\} .
\end{aligned}
$$

Proof. To investigate stability of the nonlinear time-delay system (3) consider a candidate Lyapunov-Krasovskii functional $W\left(x_{t}\right)$ as given in the formulation of the theorem, where $b>0, g>0$ and $\rho>0$ are parameters selected below, and $V$ is a homogeneous Lyapunov function for the case $\tau=0$, which exists due to Assumption 2 and satisfies (4) with $\mu>2$ if $\nu<0$ or $\mu>\nu+2$ for $\nu>0$. Let us investigate positive definiteness of $W$. Obviously $W(0)=0$ and

$$
W\left(x_{t}\right) \geq c_{1}|x(t)|_{r}^{\mu}+b \int_{t-\tau}^{t}|x(s)|_{r}^{\rho} d s-w \int_{t-\tau}^{t} \max \left\{|x(t)|_{r}^{\mu-1}|x(s)|_{r}^{\nu+1},|x(t)|_{r}^{\mu+\nu}\right\} d s,
$$

where the first property from Lemma 5 was utilized. Therefore, the following inequalities have to hold for positivity of $W$ :

$$
\begin{gathered}
c_{1}|x(t)|_{r}^{\mu}+b \int_{t-\tau}^{t}|x(s)|_{r}^{\rho} d s-w \tau|x(t)|_{r}^{\mu+\nu} \geq 0, \\
c_{1}|x(t)|_{r}^{\mu}+b \int_{t-\tau}^{t}|x(s)|_{r}^{\rho}-w|x(t)|_{r}^{\mu-1}|x(s)|_{r}^{\nu+1} d s \geq 0,
\end{gathered}
$$

and the former is valid if

$$
\max \left\{|x(t)|_{r}^{\mu}, \int_{t-\tau}^{t}|x(s)|_{r}^{\rho} d s\right\}-\frac{w \tau}{\min \left\{c_{1}, b\right\}}|x(t)|_{r}^{\mu+\nu} \geq 0
$$

and, hence, for

$$
\left\{\begin{array}{l}
\max \left\{|x(t)|_{r}^{\mu}, \int_{t-\tau}^{t}|x(s)|_{r}^{\rho} d s\right\} \geq\left(\frac{w \tau}{\min \left\{c_{1}, b\right\}}\right)^{-\frac{\mu}{\nu}} \quad \nu<0 \\
\max \left\{|x(t)|_{r}^{\mu}, \int_{t-\tau}^{t}|x(s)|_{r}^{\rho} d s\right\} \leq\left(\frac{w \tau}{\min \left\{c_{1}, b\right\}}\right)^{-\frac{\mu}{\nu}} \quad \nu>0
\end{array}\right.
$$


the first inequality is true. For the latter, using Hölder's inequality, we obtain:

$$
\begin{gathered}
\int_{t-\tau}^{t}|x(s)|_{r}^{\nu+1}|x(t)|_{r}^{\mu-1} d s \leq\left(\int_{t-\tau}^{t}|x(s)|_{r}^{\rho} d s\right)^{\frac{\nu+1}{\rho}}\left(\int_{t-\tau}^{t}|x(t)|_{r}^{\frac{\rho(\mu-1)}{\rho-\nu-1}} d s\right)^{\frac{\rho-\nu-1}{\rho}} \\
=\tau^{\frac{\rho-\nu-1}{\rho}}|x(t)|_{r}^{\mu-1}\left(\int_{t-\tau}^{t}|x(s)|_{r}^{\rho} d s\right)^{\frac{\nu+1}{\rho}} .
\end{gathered}
$$

Then for positivity of $W$ the property has to satisfy:

$$
c_{1}|x(t)|_{r}^{\mu}+b \int_{t-\tau}^{t}|x(s)|_{r}^{\rho} d s-w \tau^{\frac{\rho-\nu-1}{\rho}}|x(t)|_{r}^{\mu-1}\left(\int_{t-\tau}^{t}|x(s)|_{r}^{\rho} d s\right)^{\frac{\nu+1}{\rho}} \geq 0
$$

that is true provided the conditions of Lemma 4 are verified for $\mathfrak{a}=|x(t)|_{r}, \mathfrak{b}=\int_{t-\tau}^{t}|x(s)|{ }_{r}^{\rho} d s, \alpha=\mu, \beta=1, \gamma=\mu-1$, $\delta=\frac{\nu+1}{\rho}$ and $\ell=\frac{w \tau \frac{\rho-\nu-1}{\rho}}{\min \left\{c_{1}, b\right\}}$, which can be formulated as: $\max \left\{|x(t)|_{r}^{\mu}, \int_{t-\tau}^{t}|x(s)|_{r}^{\rho} d s\right\} \geq\left(\frac{w \tau}{\min \left\{c_{1}, b\right\}}\right)^{\frac{\rho-\nu-1}{1-\frac{\mu-1}{\mu}-\frac{\nu+1}{\rho}}}$ with $\frac{\nu+1}{\rho}<\frac{1}{\mu}$ for $\nu<0$, or $\max \left\{|x(t)|_{r}^{\mu}, \int_{t-\tau}^{t}|x(s)|_{r}^{\rho} d s\right\} \leq\left(\frac{w \tau \frac{\rho-\nu-1}{\rho}}{\min \left\{c_{1}, b\right\}}\right)^{\frac{1}{1-\frac{\mu-1}{\mu}-\frac{\nu+1}{\rho}}}$ with $\frac{\nu+1}{\rho}>\frac{1}{\mu}$ for $\nu>0$. Hence, taking $\rho>\mu(\nu+1)$ for $\nu<0$ or $\rho<\mu(\nu+1)$ for $\nu>0$ ensures positive definiteness of $W$ outside of a vicinity of the origin $\underline{\Omega}_{1}$ or locally at zero in $\bar{\Omega}_{1}$, respectively.

The derivative of the Lyapunov-Krasovskii functional $W(t)=W\left(x_{t}\right)$ with respect to time for the system (3) takes the form:

$$
\begin{gathered}
\dot{W}(t)=D^{+} W\left(x_{t}\right)=\frac{d V(x(t))}{d x(t)} F(x(t), x(t-\tau))+b\left[|x(t)|_{r}^{\rho}-|x(t-\tau)|_{r}^{\rho}\right] \\
+g\left[\tau|x(t)|_{r}^{\rho}-\int_{t-\tau}^{t}|x(s)|_{r}^{\rho} d s\right]+\frac{d V(x(t))}{d x(t)} F(x(t), x(t))-\frac{d V(x(t))}{d x(t)} F(x(t), x(t-\tau))+H\left(x_{t}\right) \\
\leq-\varsigma|x(t)|_{r}^{\nu+\mu}+(b+g \tau)|x(t)|_{r}^{\rho}-b|x(t-\tau)|_{r}^{\rho}-g \int_{t-\tau}^{t}|x(s)|_{r}^{\rho} d s+H\left(x_{t}\right),
\end{gathered}
$$

where the property (4) was used and

$$
\begin{gathered}
H\left(x_{t}\right)=\int_{t-\tau}^{t} F(x(t), x(t-\tau))^{\top} \frac{d^{2} V(x(t))}{d x(t)^{2}} F(x(t), x(s)) \\
+\frac{d V(x(t))}{d x(t)} \frac{\partial F(x(t), x(s))}{\partial x(t)} F(x(t), x(t-\tau)) d s .
\end{gathered}
$$

Following Lemma 5,

$$
\begin{aligned}
& \left|H\left(x_{t}\right)\right| \leq \int_{t-\tau}^{t} v \max \left\{|x(t)|_{r}^{\mu+2 \nu},|x(t)|_{r}^{\mu-2}|x(s)|_{r}^{2 \nu+2},|x(t)|_{r}^{\mu-2}|x(t-\tau)|_{r}^{2 \nu+2}\right\} \\
& \quad+u \max \left\{|x(t)|_{r}^{\mu+2 \nu},|x(t)|_{r}^{\frac{\mu}{2}-1}|x(t-\tau)|_{r}^{2 \nu+\frac{\mu}{2}+1},|x(t)|_{r}^{\frac{\mu}{2}-1}|x(s)|_{r}^{2 \nu+\frac{\mu}{2}+1}\right\} d s
\end{aligned}
$$

for $\nu<0$ or

$$
\begin{gathered}
\left|H\left(x_{t}\right)\right| \leq \int_{t-\tau}^{t} v \max \left\{|x(t)|_{r}^{\mu+2 \nu},|x(t)|_{r}^{\mu-2}|x(s)|_{r}^{2 \nu+2},|x(t)|_{r}^{\mu-2}|x(t-\tau)|_{r}^{2 \nu+2}\right\} \\
+u \max \left\{|x(t)|_{r}^{\mu+2 \nu},|x(t)|_{r}^{\mu-1}|x(t-\tau)|_{r}^{2 \nu+1},|x(t)|_{r}^{\mu-1}|x(s)|_{r}^{2 \nu+1}\right\} d s
\end{gathered}
$$

for $\nu>0$, and let us investigate the conditions of negative definiteness of $\dot{W}$ in the domains of positive definiteness of $W$ for different signs of degree $\nu$. First,

$$
-\frac{\varsigma}{3}|x(t)|_{r}^{\nu+\mu}-\frac{g}{3} \int_{t-\tau}^{t}|x(s)|_{r}^{\rho} d s+(b+g \tau)|x(t)|_{r}^{\rho} \leq 0
$$

that follows

$$
\max \left\{|x(t)|_{r}^{\nu+\mu}, \int_{t-\tau}^{t}|x(s)|_{r}^{\rho} d s\right\}-3 \frac{b+g \tau}{\min \{\varsigma, g\}}|x(t)|_{r}^{\rho} \geq 0
$$


and which is satisfied for $\nu<0$ provided that $\nu+\mu>\rho$ and $\max \left\{|x(t)|_{r}^{\nu+\mu}, \int_{t-\tau}^{t}|x(s)|_{r}^{\rho} d s\right\} \geq\left(3 \frac{b+g \tau}{\min \{\varsigma, g\}}\right)^{\frac{\nu+\mu}{\nu+\mu-\rho}}$; or for $\nu>0$ if $\nu+\mu<\rho$ and $\max \left\{|x(t)|_{r}^{\nu+\mu}, \int_{t-\tau}^{t}|x(s)|_{r}^{\rho} d s\right\} \leq\left(3 \frac{b+g \tau}{\min \{\varsigma, g\}}\right)^{\frac{\nu+\mu}{\nu+\mu-\rho}}$. Second,

$$
\frac{\varsigma}{3}|x(t)|_{r}^{\nu+\mu}+\frac{b}{2}|x(t-\tau)|_{r}^{\rho}+\int_{t-\tau}^{t} \frac{g}{3}|x(s)|_{r}^{\rho}-v \max \left\{|x(t)|_{r}^{\mu+2 \nu},|x(t)|_{r}^{\mu-2}|x(s)|_{r}^{2 \nu+2},|x(t)|_{r}^{\mu-2}|x(t-\tau)|_{r}^{2 \nu+2}\right\} d s \geq 0
$$

if the series of inequalities

$$
\begin{gathered}
\frac{\varsigma}{3}|x(t)|_{r}^{\nu+\mu}+\frac{g}{3} \int_{t-\tau}^{t}|x(s)|_{r}^{\rho} d s-v \tau|x(t)|_{r}^{\mu+2 \nu} \geq 0 \\
\frac{\varsigma}{3}|x(t)|_{r}^{\nu+\mu}+\frac{b}{2}|x(t-\tau)|_{r}^{\rho}+\frac{g}{3} \int_{t-\tau}^{t}|x(s)|_{r}^{\rho} d s-v \tau|x(t)|_{r}^{\mu-2}|x(t-\tau)|_{r}^{2 \nu+2} \geq 0 \\
\frac{\varsigma}{3}|x(t)|_{r}^{\nu+\mu}+\int_{t-\tau}^{t} \frac{g}{3}|x(s)|_{r}^{\rho}-v|x(t)|_{r}^{\mu-2}|x(s)|_{r}^{2 \nu+2} d s \geq 0
\end{gathered}
$$

is verified simultaneously. The first inequality follows

$$
\max \left\{|x(t)|_{r}^{\nu+\mu}, \int_{t-\tau}^{t}|x(s)|_{r}^{\rho} d s\right\}-\frac{3 v \tau}{\min \{\varsigma, g\}}|x(t)|_{r}^{\mu+2 \nu} \geq 0,
$$

then it is satisfied for $\max \left\{|x(t)|_{r}^{\nu+\mu}, \int_{t-\tau}^{t}|x(s)|_{r}^{\rho} d s\right\} \geq\left(\frac{3 v \tau}{\min \{s, g\}}\right)^{-\frac{\nu+\mu}{\nu}}$ and $\nu<0$ or $\max \left\{|x(t)|_{r}^{\nu+\mu}, \int_{t-\tau}^{t}|x(s)|_{r}^{\rho} d s\right\} \leq$ $\left(\frac{3 v \tau}{\min \{\varsigma, g\}}\right)^{-\frac{\nu+\mu}{\nu}}$ and $\nu>0$. For the second relation, by applying Young's inequality to the term $|x(t)|_{r}^{\mu-2}|x(t-\tau)|_{r}^{2(\nu+1)}$ we get:

$$
\frac{\varsigma}{3}|x(t)|_{r}^{\nu+\mu}-v \frac{\rho-2(\nu+1)}{\rho} \tau|x(t)|_{r}^{\frac{(\mu-2) \rho}{\rho-2(\nu+1)}}+\frac{g}{3} \int_{t-\tau}^{t}|x(s)|_{r}^{\rho} d s+\left(\frac{b}{2}-v \frac{2(\nu+1)}{\rho} \tau\right)|x(t-\tau)|_{r}^{\rho} \geq 0
$$

that for $\frac{b}{2} \geq v \frac{2(\nu+1)}{\rho} \tau$ follows from

$$
\max \left\{|x(t)|_{r}^{\nu+\mu}, \int_{t-\tau}^{t}|x(s)|_{r}^{\rho} d s\right\}-3 v \frac{\rho-2(\nu+1)}{\rho \min \{\varsigma, g\}} \tau|x(t)|_{r}^{\frac{(\mu-2) \rho}{\rho-2(\nu+1)}} \geq 0,
$$

which is true for $\max \left\{|x(t)|_{r}^{\nu+\mu}, \int_{t-\tau}^{t}|x(s)|_{r}^{\rho} d s\right\} \geq\left(3 v \frac{\rho-2(\nu+1)}{\rho \min \{s, g\}} \tau\right)^{\frac{(\rho-2(\nu+1))(\nu+\mu)}{(\nu+2) \rho-2(\nu+1)(\mu+\nu)}}, \rho>2(\nu+1) \frac{\mu+\nu}{\nu+2}$ and $\nu<0$, or $\max \left\{|x(t)|_{r}^{\nu+\mu}, \int_{t-\tau}^{t}|x(s)|_{r}^{\rho} d s\right\} \leq\left(3 v \frac{\rho-2(\nu+1)}{\rho \min \{s, g\}} \tau\right)^{\frac{(\rho-2(\nu+1))(\nu+\mu)}{(\nu+2) \rho-2(\nu+1)(\mu+\nu)}}, 2(\nu+1)<\rho<2(\nu+1) \frac{\mu+\nu}{\nu+2}$ and $\nu>0$. To check the third property, by applying Hölder's inequality to the term $\int_{t-\tau}^{t}|x(s)|_{r}^{2 \nu+2}|x(t)|_{r}^{\mu-2} d s$ we derive:

$$
\frac{\varsigma}{3}|x(t)|_{r}^{\nu+\mu}+\frac{g}{3} \int_{t-\tau}^{t}|x(s)|_{r}^{\rho} d s-v \tau^{\frac{\rho-2 \nu-2}{\rho}}|x(t)|_{r}^{\mu-2}\left(\int_{t-\tau}^{t}|x(s)|_{r}^{\rho} d s\right)^{\frac{2 \nu+2}{\rho}} \geq 0
$$

which is true provided the conditions of Lemma 4 are verified for $\mathfrak{a}=|x(t)|_{r}, \mathfrak{b}=\int_{t-\tau}^{t}|x(s)|{ }_{r}^{\rho} d s, \alpha=\nu+\mu, \beta=1, \gamma=\mu-2$, $\delta=\frac{2 \nu+2}{\rho}$ and $\ell=\frac{3 v \tau^{\frac{\rho-2 \nu-2}{\rho}}}{\min \{\varsigma, g\}}$, and that can be formulated as: $\max \left\{|x(t)|_{r}^{\nu+\mu}, \int_{t-\tau}^{t}|x(s)|_{r}^{\rho} d s\right\} \geq\left(\frac{3 v \tau \frac{\rho-2 \nu-2}{\rho}}{\min \{\varsigma, g\}}\right)^{\frac{1-\frac{\mu-2}{\nu+\mu}-\frac{2 \nu+2}{\rho}}{1-2}}$ with $(\nu+\mu) \frac{2 \nu+2}{\nu+2}<\rho$ for $\nu<0$, or $\max \left\{|x(t)|_{r}^{\nu+\mu}, \int_{t-\tau}^{t}|x(s)|_{r}^{\rho} d s\right\} \leq\left(\frac{3 v \tau \frac{\rho-2 \nu-2}{\rho}}{\min \{\varsigma, g\}}\right)^{\frac{1}{1-\frac{\mu-2}{\nu+\mu}-\frac{2 \nu+2}{\rho}}}$ with $(\nu+\mu) \frac{2 \nu+2}{\nu+2}>\rho$ for $\nu>0$. Finally, for $\nu<0$ the property

$\frac{\varsigma}{3}|x(t)|_{r}^{\nu+\mu}+\frac{b}{2}|x(t-\tau)|_{r}^{\rho}+\int_{t-\tau}^{t} \frac{g}{3}|x(s)|_{r}^{\rho}-u \max \left\{|x(t)|_{r}^{\mu+2 \nu},|x(t)|_{r}^{\frac{\mu}{2}-1}|x(t-\tau)|_{r}^{2 \nu+\frac{\mu}{2}+1},|x(t)|_{r}^{\frac{\mu}{2}-1}|x(s)|_{r}^{2 \nu+\frac{\mu}{2}+1}\right\} d s \geq 0$, 
follows the series of inequalities:

$$
\begin{gathered}
\frac{\varsigma}{3}|x(t)|_{r}^{\nu+\mu}+\frac{g}{3} \int_{t-\tau}^{t}|x(s)|_{r}^{\rho} d s-u \tau|x(t)|_{r}^{2 \nu+\mu} \geq 0 \\
\frac{\varsigma}{3}|x(t)|_{r}^{\nu+\mu}+\frac{b}{2}|x(t-\tau)|_{r}^{\rho}+\frac{g}{3} \int_{t-\tau}^{t}|x(s)|{ }_{r}^{\rho} d s-u \tau|x(t)|_{r}^{\frac{\mu}{2}-1}|x(t-\tau)|_{r}^{2 \nu+\frac{\mu}{2}+1} \geq 0 \\
\frac{\varsigma}{3}|x(t)|_{r}^{\nu+\mu}+\int_{t-\tau}^{t} \frac{g}{3}|x(s)|_{r}^{\rho}-u|x(t)|_{r}^{\frac{\mu}{2}-1}|x(s)|_{r}^{2 \nu+\frac{\mu}{2}+1} d s \geq 0 .
\end{gathered}
$$

The first inequality can be treated directly, and it is satisfied for $\max \left\{|x(t)|_{r}^{\nu+\mu}, \int_{t-\tau}^{t}|x(s)|_{r}^{\rho} d s\right\} \geq\left(\frac{3 u \tau}{\min \{s, g\}}\right)^{-\frac{\nu+\mu}{\nu}}$. For the second case, applying Young's inequality we obtain

$$
\frac{\varsigma}{3}|x(t)|_{r}^{\nu+\mu}-u \tau \frac{\rho-\frac{\mu}{2}-2 \nu-1}{\rho}|x(t)|_{r}^{\frac{\left(\frac{\mu}{2}-1\right) \rho}{\rho-\frac{\mu}{2}-2 \nu-1}}+\frac{g}{3} \int_{t-\tau}^{t}|x(s)|_{r}^{\rho} d s+\left(\frac{b}{2}-u \frac{\frac{\mu}{2}+2 \nu+1}{\rho} \tau\right)|x(t-\tau)|_{r}^{\rho} \geq 0
$$

that for $\frac{b}{2} \geq u \frac{\frac{\mu}{2}+2 \nu+1}{\rho} \tau$ it is followed by

$$
\max \left\{|x(t)|_{r}^{\nu+\mu}, \int_{t-\tau}^{t}|x(s)|_{r}^{\rho} d s\right\}-3 u \frac{\rho-\frac{\mu}{2}-2 \nu-1}{\rho \min \{\varsigma, g\}} \tau|x(t)|_{r}^{\frac{\left(\frac{\mu}{2}-1\right) \rho}{\rho-\frac{\mu}{2}-2 \nu-1}} \geq 0,
$$

which is true for

$$
\max \left\{|x(t)|_{r}^{\nu+\mu}, \int_{t-\tau}^{t}|x(s)|_{r}^{\rho} d s\right\} \geq\left(3 u \frac{\rho-\frac{\mu}{2}-2 \nu-1}{\rho \min \{\varsigma, g\}} \tau\right)^{\frac{\left(\rho-\frac{\mu}{2}-2 \nu-1\right)(\nu+\mu)}{(\nu+\mu)\left(\rho-\frac{\mu}{2}-2 \nu-1\right)-\left(\frac{\mu}{2}-1\right) \rho}}
$$

and $\rho>\max \left\{\frac{\mu}{2}+2 \nu+1, \frac{2\left(\nu+\frac{\mu}{2}\right)^{2}+\nu+\left(\frac{\nu}{2}+1\right) \mu}{\nu+1+\frac{\mu}{2}}\right\}$. Finally, for the third property using Hölder's inequality, we get

$$
\frac{\varsigma}{3}|x(t)|_{r}^{\nu+\mu}+\frac{g}{3} \int_{t-\tau}^{t}|x(s)|_{r}^{\rho} d s-u \tau^{\frac{\rho-\frac{\mu}{2}-2 \nu-1}{\rho}}|x(t)|_{r}^{\frac{\mu}{2}-1}\left(\int_{t-\tau}^{t}|x(s)|_{r}^{\rho} d s\right)^{\frac{\frac{\mu}{2}+2 \nu+1}{\rho}} \geq 0
$$

which is true provided the conditions of Lemma 4 are verified for $\mathfrak{a}=|x(t)|_{r}, \mathfrak{b}=\int_{t-\tau}^{t}|x(s)|_{r}^{\rho} d s, \alpha=\nu+\mu, \beta=1$, $\gamma=\frac{\mu}{2}-1, \delta=\frac{\frac{\mu}{2}+2 \nu+1}{\rho}$ and $\ell=\frac{3 u \tau \frac{\rho-\frac{\mu}{2}-2 \nu-1}{\rho}}{\min \{\varsigma, g\}}$, and that can be formulated as:

$$
\max \left\{|x(t)|_{r}^{\nu+\mu}, \int_{t-\tau}^{t}|x(s)|_{r}^{\rho} d s\right\} \geq\left(\frac{3 u \tau^{\frac{\rho-\frac{\mu}{2}-2 \nu-1}{\rho}}}{\min \{\varsigma, g\}}\right)^{\frac{1}{1-\frac{\mu}{2}-1} \frac{\mu}{\nu+\mu}-\frac{\frac{\mu}{2}+2 \nu+1}{\rho}}
$$

with $(\nu+\mu) \frac{\frac{\mu}{2}+2 \nu+1}{\nu+\frac{\mu}{2}+1}<\rho$. For $\nu>0$ the property

$$
\frac{\varsigma}{3}|x(t)|_{r}^{\nu+\mu}+\frac{b}{2}|x(t-\tau)|_{r}^{\rho}+\int_{t-\tau}^{t} \frac{g}{3}|x(s)|_{r}^{\rho}-u \max \left\{|x(t)|_{r}^{\mu+2 \nu},|x(t)|_{r}^{\mu-1}|x(t-\tau)|_{r}^{2 \nu+1},|x(t)|_{r}^{\mu-1}|x(s)|_{r}^{2 \nu+1}\right\} d s \geq 0,
$$

follows the series of inequalities:

$$
\begin{gathered}
\frac{\varsigma}{3}|x(t)|_{r}^{\nu+\mu}+\frac{g}{3} \int_{t-\tau}^{t}|x(s)|_{r}^{\rho} d s-u \tau|x(t)|_{r}^{2 \nu+\mu} \geq 0 \\
\frac{\varsigma}{3}|x(t)|_{r}^{\nu+\mu}+\frac{b}{2}|x(t-\tau)|_{r}^{\rho}+\frac{g}{3} \int_{t-\tau}^{t}|x(s)|_{r}^{\rho} d s-u \tau|x(t)|_{r}^{\mu-1}|x(t-\tau)|_{r}^{2 \nu+1} \geq 0 \\
\frac{\varsigma}{3}|x(t)|_{r}^{\nu+\mu}+\int_{t-\tau}^{t} \frac{g}{3}|x(s)|_{r}^{\rho}-u|x(t)|_{r}^{\mu-1}|x(s)|_{r}^{2 \nu+1} d s \geq 0 .
\end{gathered}
$$

The first inequality as before is satisfied for $\max \left\{|x(t)|_{r}^{\nu+\mu}, \int_{t-\tau}^{t}|x(s)|_{r}^{\rho} d s\right\} \leq\left(\frac{3 u \tau}{\min \{\varsigma, g\}}\right)^{-\frac{\nu+\mu}{\nu}}$. For the second case, applying 
Young's inequality we obtain

$$
\frac{\varsigma}{3}|x(t)|_{r}^{\nu+\mu}-u \tau \frac{\rho-2 \nu-1}{\rho}|x(t)|_{r}^{\frac{(\mu-1) \rho}{\rho-2 \nu-1}}+\frac{g}{3} \int_{t-\tau}^{t}|x(s)|_{r}^{\rho} d s+\left(\frac{b}{2}-u \frac{2 \nu+1}{\rho} \tau\right)|x(t-\tau)|_{r}^{\rho} \geq 0
$$

that for $\frac{b}{2} \geq u \frac{2 \nu+1}{\rho} \tau$ is followed by

$$
\max \left\{|x(t)|_{r}^{\nu+\mu}, \int_{t-\tau}^{t}|x(s)|_{r}^{\rho} d s\right\}-3 u \frac{\rho-2 \nu-1}{\rho \min \{\varsigma, g\}} \tau|x(t)|_{r}^{\frac{(\mu-1) \rho}{\rho-2 \nu-1}} \geq 0
$$

which is true for $\max \left\{|x(t)|_{r}^{\nu+\mu}, \int_{t-\tau}^{t}|x(s)|_{r}^{\rho} d s\right\} \leq\left(3 u \frac{\rho-2 \nu-1}{\rho \min \{s, g\}} \tau\right)^{\frac{(\rho-2 \nu-1)(\nu+\mu)}{(\nu+1) \rho-(2 \nu+1)(\nu+\mu)}}, 2 \nu+1<\rho<\frac{(2 \nu+1)(\nu+\mu)}{\nu+1}$. Finally, for the third case using Hölder's inequality, we get

$$
\frac{\varsigma}{3}|x(t)|_{r}^{\nu+\mu}+\frac{g}{3} \int_{t-\tau}^{t}|x(s)|_{r}^{\rho} d s-u \tau^{\frac{\rho-2 \nu-1}{\rho}}|x(t)|_{r}^{\mu-1}\left(\int_{t-\tau}^{t}|x(s)|_{r}^{\rho} d s\right)^{\frac{2 \nu+1}{\rho}} \geq 0,
$$

which is true provided the conditions of Lemma 4 are verified for $\mathfrak{a}=|x(t)|_{r}, \mathfrak{b}=\int_{t-\tau}^{t} \mid x(s){ }_{r}^{\rho} d s, \alpha=\nu+\mu, \beta=1, \gamma=\mu-1$, $\delta=\frac{2 \nu+1}{\rho}$ and $\ell=\frac{3 u \tau \frac{\rho-2 \nu-1}{\rho}}{\min \{\varsigma, g\}}$, and that can be formulated as $\max \left\{|x(t)|_{r}^{\nu+\mu}, \int_{t-\tau}^{t}|x(s)|_{r}^{\rho} d s\right\} \leq\left(\frac{3 u \tau \frac{\rho-2 \nu-1}{\rho}}{\min \{\varsigma, g\}}\right)^{\frac{\frac{\nu+1}{\nu+\mu}-\frac{2 \nu+1}{\rho}}{2}}$ with $\frac{(2 \nu+1)(\nu+\mu)}{\nu+1}>\rho$. Therefore, we established the conditions that

$$
\dot{W}(t)<0
$$

for $x_{t} \in \underline{\Omega}_{2}$ with $\nu<0$ or for $x_{t} \in \bar{\Omega}_{2}$ with $\nu>0$.

Hence, the parameters of the Lyapunov-Krasovskii functional have to respect the constraints:

$$
b>\frac{\tau}{\rho} \max \{4 v(\nu+1), u(\mu+4 \nu+2)\}, g>0 .
$$

Next, taking into account the admissible set of values for $\mu$, the restrictions imposed on $\rho$ can be summarized as follows for $\nu<0$ :

$$
\max \left\{\mu(\nu+1), 2(\nu+1) \frac{\mu+\nu}{\nu+2}, \frac{2\left(\nu+\frac{\mu}{2}\right)^{2}+\nu+\left(\frac{\nu}{2}+1\right) \mu}{\nu+1+\frac{\mu}{2}},(\nu+\mu) \frac{\frac{\mu}{2}+2 \nu+1}{\nu+\frac{\mu}{2}+1}\right\}<\rho<\nu+\mu,
$$

or for $\nu>0$ :

$$
\max \{\nu+\mu, 2(\nu+1)\}<\rho<\min \left\{\mu(\nu+1), 2(\nu+1) \frac{\mu+\nu}{\nu+2}, \frac{(2 \nu+1)(\nu+\mu)}{\nu+1}\right\} .
$$

It is straightforward to check that for both signs of degree $\nu$ there is a non-empty interval of admissible values for $\rho$ under the given restrictions on $\mu$. Consequently, the properties

$$
W\left(x_{t}\right)>0, D^{+} W\left(x_{t}\right)<0
$$

are obtained for $x_{t} \in \underline{\Omega}$ with $\nu<0$ or for $x_{t} \in \bar{\Omega}$ with $\nu>0$, where the expressions for the sets $\underline{\Omega}, \bar{\Omega}$ are given in the formulation of the theorem.

Note that $V$ can be just twice differentiable having bounded $\frac{d^{2} V(y)}{d y^{2}}$ for $y \in \mathbb{S}_{r}$ (this property is used to calculate $v$ in Lemma 5).

Remark 2. The local convergence to the origin in the case of $\nu>0$ or the global convergence to its neighborhood for $\nu<0$ follows from the result of Theorem 1 using the standard Lyapunov or Lyapunov-Krasovskii arguments [32], [2], [33].

Qualitatively, this theorem confirms the results of [27], [34], [13], [20], but it also has some quantitative estimates on the domains of stability (which are included in the sets $\underline{\Omega}$ and $\bar{\Omega}$ ) and gives the explicit relations between all parameters. Contrarily [13], [20], there is no requirement on continuity of $F$ with respect to both arguments in our results. In addition, a universal expression $W\left(x_{t}\right)$ of a Lyapunov-Krasovskii functional is presented, which can be used for any homogeneous systems of non-zero degree (it also suits for ones admitting local homogeneous approximations [31], [12]), while the estimates derived in 
the proof of Theorem 1 can be strengthened as

$$
\begin{gathered}
\underline{\alpha}\left(|x(t)|_{r}^{\mu}+\int_{t-\tau}^{t}|x(s)|_{r}^{\rho} d s\right) \leq W\left(x_{t}\right) \leq \bar{\alpha}\left(|x(t)|_{r}^{\mu}+\int_{t-\tau}^{t}|x(s)|_{r}^{\rho} d s\right), \\
\dot{W}(t) \leq-\alpha\left(|x(t)|_{r}^{\mu+\nu}+|x(t-\tau)|_{r}^{\rho}+\int_{t-\tau}^{t}|x(s)|_{r}^{\rho} d s\right)
\end{gathered}
$$

for all $x_{t} \in \underline{\Omega}$ with $\nu<0$ or for all $x_{t} \in \bar{\Omega}$ with $\nu>0$, for suitably defined $0<\underline{\alpha} \leq \bar{\alpha}$ and $\alpha>0$, which is equivalent to

$$
\dot{W} \leq-\tilde{\alpha} W^{1+\frac{\nu}{\mu}}
$$

for some $\tilde{\alpha}>0$ dependent on $\underline{\Omega}$ or $\bar{\Omega}$. Note that

$$
\max \left\{|x(t)|_{r}^{\nu+\mu}, \int_{t-\tau}^{t}|x(s)|_{r}^{\rho} d s\right\} \leq \max \{1, \tau\} \max \left\{\left\|x_{t}\right\|_{r}^{\nu+\mu},\left\|x_{t}\right\|_{r}^{\rho}\right\}
$$

then the set $\bar{\Omega}$ can be re-formulated using the standard norm. Similarly, $\left\|x_{t}\right\|=|x(t-\theta)|$ for some $\theta \in[-\tau, 0]$, then

$$
\begin{gathered}
|x(t-\theta)|=\left|x(t)-\int_{t-\theta}^{t} \dot{x}(s) d s\right| \leq|x(t)|+\int_{t-\tau}^{t}|\dot{x}(s)| d s \\
\leq \bar{\sigma}_{r}\left(|x(t)|_{r}\right)+\int_{t-\tau}^{t} \bar{\sigma}_{r}\left(|F(x(s), x(s-\tau))|_{r}\right) d s,
\end{gathered}
$$

next defining $\kappa=\sup _{|\xi|_{r} \leq 1,|\zeta|_{r} \leq 1}|F(\xi, \zeta)|_{r}$ and $\lambda=\max \left\{|x(s)|_{r},|x(s-\tau)|_{r}\right\}$ we obtain:

$$
\begin{gathered}
\left\|x_{t}\right\| \leq \bar{\sigma}_{r}\left(|x(t)|_{r}\right)+\int_{t-\tau}^{t} \bar{\sigma}_{r}\left(\kappa \max \left\{\lambda^{1+r_{\min }^{-1}}, \lambda^{1+r_{\max }^{-1}}\right\}\right) d s \\
\leq \bar{\sigma}_{r}\left(|x(t)|_{r}\right)+\int_{t-2 \tau}^{t} \bar{\sigma}_{r}\left(2 \kappa\left(2|x(s)|_{r}\right)^{1+r_{\min }^{-1}}\right)+\bar{\sigma}_{r}\left(2 \kappa\left(2|x(s)|_{r}\right)^{1+r_{\max }^{-1}}\right) d s,
\end{gathered}
$$

which implies that for an extended space $C_{[-2 \tau, 0]}$ the set $\underline{\Omega}$ also can be presented using the conventional norm.

Remark 3. It is important to highlight, that the parameter $b$ of the Lyapunov-Krasovskii functional $W$ can be selected proportionally to $\max \left\{\tau, \tau^{\omega}\right\}$ with $\omega<\frac{\rho-\nu-1}{\rho}$, then the domain of convergence estimated in $\underline{\Omega}, \bar{\Omega}$ becomes also proportional to $\tau$. In other words, the obtained estimates imply that when the value of delay $\tau$ is approaching zero, the domain of convergence to the origin for $\nu>0$ grows to infinity, while the zone globally attracting the trajectories for $\nu<0$ shrinks to the origin.

Remark 4. Denote $\mathbf{1}=[1, \ldots, 1]^{\top}$, then $\mathbf{1}$-homogeneous systems are homogeneous in the standard (Euler) sense. The result of Theorem 1 in the case of standard homogeneity and positive degree was obtained in [28]. Nevertheless, the result proven in Theorem 1 for the case of weighted homogeneity has sense, since a transformation of $\mathbf{r}$-homogeneous dynamics to the corresponding 1-homogeneous can be tricky. Indeed, there exist transformations of coordinates making an $\mathbf{r}-$ homogeneous system 1-homogeneous [35], e.g.,

$$
y_{i}=\left|x_{i}\right|^{q r_{i}^{-1}} \operatorname{sign}\left(x_{i}\right), i=\overline{1, n} .
$$

However, such a transformation may not preserve stability properties of (3). For an example, consider a double integrator stabilized by a delayed feedback that satisfies the conditions of assumptions 1 and 2 for $\mathbf{r}=[1,1+\nu]$ with the degree $\nu \in[-0.5,0):$

$$
\begin{gathered}
\dot{x}_{1}(t)=x_{2}(t), \\
\dot{x}_{2}(t)=-k_{1}\left|x_{1}(t-\tau)\right|^{1+2 \nu} \operatorname{sign}\left(x_{1}(t-\tau)\right)-k_{2}\left|x_{2}(t-\tau)\right|^{\frac{1+2 \nu}{1+\nu}} \operatorname{sign}\left(x_{2}(t-\tau)\right) .
\end{gathered}
$$

The derivative of $F$ with respect to the first (non-delayed) argument is well-defined. Consider a change of coordinates:

$$
y_{1}=x_{1}, y_{2}=\left|x_{2}\right|^{\frac{1}{1+\nu}} \operatorname{sign}\left(x_{2}\right),
$$


then in the new coordinates we obtain:

$$
\begin{gathered}
\dot{y}_{1}(t)=\left|y_{2}(t)\right|^{1+\nu} \operatorname{sign}\left(y_{2}(t)\right), \\
\dot{y}_{2}(t)=-\left|y_{2}(t)\right|^{-\nu} \frac{k_{1}}{1+\nu}\left|y_{1}(t-\tau)\right|^{1+2 \nu} \operatorname{sign}\left(y_{1}(t-\tau)\right)-\left|y_{2}(t)\right|^{-\nu} \frac{k_{2}}{1+\nu}\left|y_{2}(t-\tau)\right|^{1+2 \nu} \operatorname{sign}\left(y_{2}(t-\tau)\right),
\end{gathered}
$$

which is 1 -homogeneous of degree $1+\nu$, but it has a continuum of equilibria on the line $y_{2}=0$ (a change of the time $\tilde{t}=\int_{0}^{t}\left|y_{2}(s)\right|^{-\nu} d s$ is hard to apply here due to the presence of delay).

The requirement on boundedness of the derivative of $F$ with respect to the first argument (imposed in Assumption 1) can be relaxed by considering a special form of the system:

Corollary 1. Let $F(x, z)=F_{1}(x)+F_{2}(z)$ for some functions $F_{1}, F_{2}: \mathbb{R}^{n} \rightarrow \mathbb{R}^{n}$, the system (3) be $\mathbf{r}$-homogeneous of degree $\nu \geq-r_{\min }, \sup _{|\xi|_{r} \leq 1,|\zeta|_{r} \leq 1}|F(\xi, \zeta)|<+\infty$ and Assumption 2 hold. Then for any value of the delay $\tau>0$ the properties

$$
W\left(x_{t}\right)>0, D^{+} W\left(x_{t}\right)<0
$$

are satisfied for $x_{t} \in \underline{\Omega}$ if $\nu<0$ or for $x_{t} \in \bar{\Omega}$ if $\nu>0$, where

$$
\begin{aligned}
W\left(x_{t}\right)= & V(x(t))+b \int_{t-\tau}^{t}|x(s)|_{r}^{\rho} d s+g \int_{t-\tau}^{t}(s-t+\tau)|x(s)|_{r}^{\rho} d s \\
& +\int_{t-\tau}^{t} \frac{d V(x(t))}{d x} F_{2}(x(s)) d s,
\end{aligned}
$$

is a Lyapunov-Krasovskii functional for (3); the meaning and constraints imposed on parameters $b, g$ and $\rho$ are the same as in Theorem 1 under substitution $u=0$.

Proof. The proof follows exactly the steps of the proof of Theorem 1 taking into account that

$$
H\left(x_{t}\right)=\int_{t-\tau}^{t} F(x(t), x(t-\tau))^{\top} \frac{d^{2} V(x(t))}{d x(t)^{2}} F_{2}(x(s)) d s
$$

and there is no term proportional to $u$ in the analysis.

Let us demonstrate applicability of the presented results on a real case.

\section{APPLICATION}

In the paper [36], for a super-twisting differentiation/observation algorithm,

$$
\begin{aligned}
& \dot{x}_{1}(t)=-k_{1} \sqrt{\left|x_{1}(t)\right|} \operatorname{sign}\left(x_{1}(t)\right)+x_{2}(t), \\
& \dot{x}_{2}(t)=-k_{2} \operatorname{sign}\left(x_{1}(t)\right),
\end{aligned}
$$

$x(t)=\left[x_{1}(t), x_{2}(t)\right]^{\top} \in \mathbb{R}^{2}$, where $0<k_{2}<k_{1}<+\infty$ are the observer gains, the following Lyapunov function has been proposed:

$$
V(x)=\eta_{1} x_{1}^{2}+\eta_{2} x_{1} x_{2}\left|x_{2}\right|+\eta_{3} x_{2}^{4}
$$

for $\eta_{1}, \eta_{3} \in \mathbb{R}_{+}$and $\eta_{2} \in \mathbb{R}$, which is continuously differentiable, and its second derivative is discontinuous (it contains $\operatorname{sign}\left(x_{2}\right)$ ) but bounded on any compact set, hence, the constant $v$ can be estimated. It is straightforward to check that the system and the Lyapunov function are $\mathbf{r}$-homogeneous of degrees $\nu=-1$ and $\mu=4$, respectively, with $\mathbf{r}=[2,1]^{\top}$. Assume that the actual output injection exhibits a delay $\tau>0$ :

$$
\begin{aligned}
& \dot{x}_{1}(t)=-k_{1} \sqrt{\left|x_{1}(t-\tau)\right|} \operatorname{sign}\left(x_{1}(t-\tau)\right)+x_{2}(t), \\
& \dot{x}_{2}(t)=-k_{2} \operatorname{sign}\left(x_{1}(t-\tau)\right),
\end{aligned}
$$

then all conditions of Corollary 1 are satisfied, and such a $F(x(t), x(t-\tau))$ is continuously differentiable with respect to the first argument. 


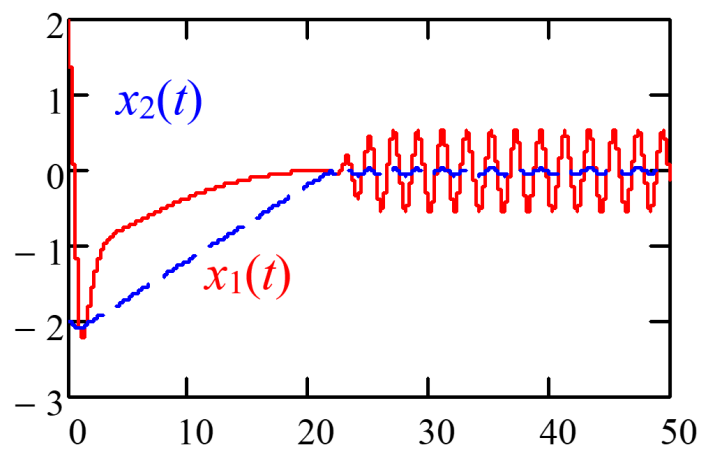

Figure 1. Behavior of trajectories of the system

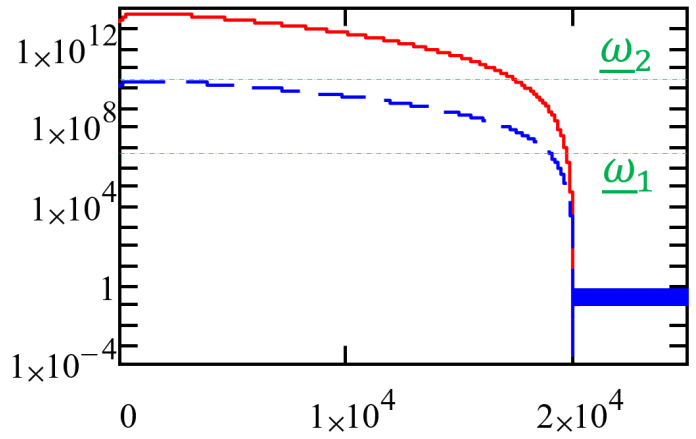

Figure 2. Behavior of norms on the trajectories of the system

Let

$$
\tau=0.5, k_{1}=2, k_{2}=0.1
$$

then according to the procedure of selection of parameters of the Lyapunov function given in [36]:

$$
\eta_{1}=4, \eta_{2}=-2, \eta_{3}=5
$$

The admissible interval for $\rho$ is $[1.5,3]$, and let $\rho=2.25$. For the norm $|x|_{r}=\sqrt{\left|x_{1}\right|}+\left|x_{2}\right|$, using a grid with 200 points on $\mathbb{S}_{r}$ we compute:

$$
c_{1}=0.434, \varsigma=0.411, w=u=42.62, v=411.616 .
$$

Take $g=10$ and $b=19>\frac{\tau}{\rho} \max \{4 v(\nu+1), u(\mu+4 \nu+2)\}$ to respect the restriction of the corollary, then finally we get:

$$
\underline{\varpi}_{1}=5.838 \times 10^{6}, \underline{\varpi}_{2}=2.934 \times 10^{10} .
$$

An example of the system trajectory is shown in Fig. 1, where behavior of $x_{1}(t)$ and $x_{2}(t)$ are depicted using red solid and blue dash lines, respectively. The system is converging to a limit cycle. An example of the comportment of the norms $y(t)=\max \left\{|x(t)|_{r}^{\nu}, \int_{t-\tau}^{t}|x(s)|_{r}^{\rho} d s\right\}$ and $\psi(t)=\max \left\{|x(t)|_{r}^{\nu+\mu}, \int_{t-\tau}^{t}|x(s)|_{r}^{\rho} d s\right\}$ are presented using red solid and blue dash lines, respectively, in Fig. 2. As we can conclude from these results of simulation, the obtained estimates are rather moderate, and for a concrete example the methodology has to be properly adjusted to reduce the conservativeness.

\section{Conclusions}

Considering the homogeneous systems with non-zero degree, which are asymptotically stable without delay, it is proposed a corresponding generic structure of a Lyapunov-Krasovskii functional, which allows also evaluation of the domain of convergence in the presence of any constant delay. In particular, we show that for the systems with a positive degree, the domain of convergence to the origin is inversely proportional to the value of the delay; while for the system with a negative degree, the size of the vicinity of the origin, which globally attracts all trajectories, is proportional to the value of delay. This Lyapunov- 
Krasovskii functional can be useful for analysis of convergence of discretization of solutions of homogeneous delayed nonlinear systems by using Euler method, which is a direction of future research.

\section{REFERENCES}

[1] K. Gu, K. Kharitonov, and J. Chen, Stability of Time-Delay Systems. Control Engineering, Boston: Birkhäuser, 2003.

[2] J. Hale, Theory of Functional Differential Equations. Springer-Verlag, 1977.

[3] V. Kolmanovsky and V. Nosov, Stability of functional differential equations. San Diego: CA: Academic, 1986.

[4] E. Fridman, Introduction to Time-Delay Systems: Analysis and Control. Basel: Birkhäuser, 2014.

[5] J.-P. Richard, "Time-delay systems: an overview of some recent advances and open problems," Automatica, vol. 39, pp. 1667-1694, 2003.

[6] E. Bernuau, D. Efimov, W. Perruquetti, and A. Polyakov, "On homogeneity and its application in sliding mode," Journal of the Franklin Institute, vol. 351, no. 4, pp. 1866-1901, 2014.

[7] A. Bacciotti and L. Rosier, Liapunov Functions and Stability in Control Theory, vol. 267 of Lecture Notes in Control and Inform. Sci. Berlin: Springer, 2001.

[8] S. Bhat and D. Bernstein, "Geometric homogeneity with applications to finite-time stability," Mathematics of Control, Signals and Systems, vol. 17, pp. 101-127, 2005.

[9] M. Kawski, Homogeneous feedback stabilization, vol. 7 of Progress in systems and control theory: New trends in systems theory. Birkhäuser, 1991.

[10] V. Zubov, "On systems of ordinary differential equations with generalized homogenous right-hand sides," Izvestia vuzov. Mathematica., vol. 1, pp. 80-88, 1958. in Russian.

[11] A. Levant, "Homogeneity approach to high-order sliding mode design," Automatica, vol. 41, no. 5, pp. 823-830, 2005.

[12] D. Efimov, W. Perruquetti, and J.-P. Richard, "Development of homogeneity concept for time-delay systems," SIAM J. Control Optim., vol. 52, no. 3, pp. 1403-1808, 2014.

[13] D. Efimov, A. Polyakov, W. Perruquetti, and J.-P. Richard, "Weighted homogeneity for time-delay systems: Finite-time and independent of delay stability," IEEE Trans. Automatic Control, vol. 61, no. 1, pp. 210-215, 2016.

[14] A. Polyakov, D. Efimov, E. Fridman, and W. Perruquetti, "On homogeneous distributed parameter systems," IEEE Trans. Automatic Control, vol. 61, no. 11 , pp. $3657-3662,2016$.

[15] L. Rosier, "Homogeneous Lyapunov function for homogeneous continuous vector field," Systems\&Control Lett., vol. 19, pp. 467-473, 1992.

[16] D. Efimov and W. Perruquetti, "Oscillations conditions in homogenous systems," in Proc. NOLCOS'10, (Bologna), pp. 1379-1384, 2010.

[17] D. Efimov, W. Perruquetti, and M. Petreczky, "On necessary conditions of instability and design of destabilizing controls," in Proc. 53rd IEEE CDC, (LA), 2014

[18] D. Efimov, R. Ushirobira, J. Moreno, and W. Perruquetti, "Homogeneous lyapunov functions: from converse design to numerical implementation," SIAM J. Control Optimization, vol. 56, no. 5, pp. 3454-3477, 2018.

[19] E. Bernuau, A. Polyakov, D. Efimov, and W. Perruquetti, "Verification of iss, iiss and ioss properties applying weighted homogeneity," Systems \& Control Letters, vol. 62, no. 12, pp. 1159-1167, 2013.

[20] K. Zimenko, D. Efimov, A. Polyakov, and W. Perruquetti, "A note on delay robustness for homogeneous systems with negative degree.," Automatica, vol. 79, no. 5, pp. 178-184, 2017.

[21] V. Andrieu, L. Praly, and A. Astolfi, "Homogeneous approximation, recursive observer design, and output feedback," SIAM J. Control Optimization, vol. 47, no. 4, pp. 1814-1850, 2008.

[22] D. Efimov and W. Perruquetti, "Conditions of oscillations and multi-homogeneity," Mathematics of Control, Signals, and Systems, vol. 28, no. 3, pp. 1-37, 2016.

[23] J. Diblik, "Asymptotic equilibrium for homogeneous delay linear differential equations with 1-perturbation term," Nonlinear Analysis, Theory, Methods \& Applicarions, vol. 30, no. 6, pp. 3927-3933, 1997.

[24] F. Asl and A. Ulsoy, "Analytical solution of a system of homogeneous delay differential equations via the Lambert function," in Proc. American Control Conference, (Chicago), pp. 2496-2500, 2000.

[25] F. Mazenc, S. Mondie, and S.-I. Niculescu, "Global asymptotic stabilization for chains of integrators with a delay in the input," in Proc. 40th IEEE Conference on Decision and Control, vol. 2, pp. 1843-1848, 2001.

[26] V. Bokharaie, O. Mason, and M. Verwoerd, "D-stability and delay-independent stability of homogeneous cooperative systems," IEEE Trans. Automatic Control, vol. 55, no. 12, pp. 2882-2885, 2010.

[27] A. Aleksandrov and A. Zhabko, "On the asymptotic stability of solutions of nonlinear systems with delay," Siberian Mathematical Journal, vol. 53, no. 3, pp. 393-403, 2012.

[28] A. Aleksandrov, A. Zhabko, and V. Pecherskiy, "Complete type functionals for some classes of homogeneous diffrential-difference systems," in Proc. 8th international conference "Modern methods of applied mathematics, control theory and computer technology", (Voronezh), pp. 5-8, 2015. in Russian.

[29] E. Fridman, L. Fridman, and E. Shustin, "Steady modes in relay control systems with time delay and periodic disturbances," Journal of Dynamic Systems, Measurement, and Control, vol. 122, no. 4, pp. 732-737, 2000.

[30] E. Shustin, E. Fridman, and L. Fridman, "Oscillations in a second-order discontinuous system with delay," Discrete \& Continuous Dynamical Systems - A, vol. 9, no. 2, pp. 339-358, 2003.

[31] D. Efimov and W. Perruquetti, "Homogeneity for time-delay systems," in Proc. IFAC WC 2011, (Milan), 2011.

[32] Y. Lin, E. Sontag, and Y. Wang, "A smooth converse Lyapunov theorem for robust stability," SIAM J. Control Optimization, vol. 34, no. 1, pp. 124-160, 1996.

[33] E. Fridman, M. Dambrine, and N. Yeganefar, "On input-to-state stability of systems with time-delay: A matrix inequalities approach," Automatica, vol. 44, no. 9, pp. 2364-2369, 2008. 
[34] A. Aleksandrov and A. Zhabko, "Delay-independent stability of homogeneous systems," Applied Mathematics Letters, vol. 34, no. 8, pp. 43-50, 2014.

[35] A. Korchak, "Design of generalized homogeneous Lyapunov-Krasovskii functions," Latvian mathematical annual journal, vol. 24, pp. 105-112, 1980.

[36] T. S' anchez and J. Moreno, "A constructive Lyapunov function design method for a class of homogeneous systems," in Proc. 53rd IEEE Conference on Decision and Control, pp. 5500-5505, 2014. 